\title{
Exercise via Videoconferencing for People with Severe Mental Illness during COVID-19 Times: A Feasibility Study
}

\author{
Lisanne Elisabeth Maria Koomen ${ }^{1,2}$, Patricia Velasquez³, Fabio D'Agata4, Jeroen Deenik1,5,6, \\ Wiepke Cahn ${ }^{1}$
}

${ }^{1}$ UMC Utrecht, Heidelberglaan, Utrecht, The Netherlands

${ }^{2}$ Lister, Utrecht, The Netherlands

${ }^{3}$ Studio Patricia, Weg naar Rhijnauwen, Utrecht, The Netherlands

${ }^{4}$ Spat Verandert, P.C. Borstraat, The Netherlands

${ }^{5}$ Maastricht University, Maastricht, The Netherlands

${ }^{6} \mathrm{GGz}$ Centraal, Amersfoort, The Netherlands

Email: *1.e.m.koomen-2@umcutrecht.nl, info@studiopatricia.com, fabio@spatverandert.nl, j.deenik@ggzcentraal.nl,

wcahn@umcutrecht.nl

How to cite this paper: Koomen, L. E. M., Velasquez, P., D’Agata, F., Deenik, J., \& Cahn, W. (2021). Exercise via Videoconferencing for People with Severe Mental Illness during COVID-19 Times: A Feasibility Study. Advances in Physical Education, 11, 239-245.

https://doi.org/10.4236/ape.2021.112019

Received: February 10, 2021

Accepted: May 11, 2021

Published: May 14, 2021

Copyright $\odot 2021$ by author(s) and Scientific Research Publishing Inc. This work is licensed under the Creative Commons Attribution International License (CC BY 4.0).

http://creativecommons.org/licenses/by/4.0/

\begin{abstract}
The COVID-19 pandemic has serious negative consequences for the health status of people with severe mental illness (SMI). In this non-randomized controlled prospective patient preference study $(\mathrm{N}=40)$, we investigated the feasibility of exercise via live videoconferencing for people with SMI, and preliminary effects on symptom severity and quality of life. We found that group exercise via videoconferencing is feasible for people with SMI, improving physical and psychological quality of life compared to controls receiving usual care. Further research in larger samples is needed to study the potential effect of exercise via videoconferencing in mitigating the negative effects of the COVID-19 pandemic on mental and physical health for people with SMI.
\end{abstract}

\section{Keywords}

COVID-19, Exercise, Severe Mental Illness, Videoconferencing

\section{Introduction}

The COVID-19 pandemic has changed our society drastically, impacting patients with severe mental illness (SMI) even more (O'Connor et al., 2020; Vindegaard \& Benros, 2020). The urgent request to stay at home worsens their already high levels of sedentary behavior in people with SMI (Stanton et al., 2020; Vancampfort et al., 2017). As higher levels of sedentary behavior and lack of 
physical activity both are associated with poorer mental and physical health (Vancampfort et al., 2017), this might contribute to a deterioration in the health status of people with SMI. It is therefore urgently needed that we take measures to decrease the negative effects on mental and physical wellbeing of the COVID-19 pandemic for patients with SMI. Physical activity can have a positive effect on psychiatric symptoms, quality of life and physical health in people with a severe mental illness (Firth et al., 2020) and might be of use to mitigate the negative effects of the pandemic. However, the mandatory governmental COVID-19 measures made regular exercise activities harder or impossible since all sports facilities were closed and people were advised to stay at home.

Therefore, the aim of this study was to set up a videoconferencing exercise intervention for people with SMI to investigate whether it is feasible for them to join in a videoconferencing exercise intervention during the COVID-19 pandemic. Additionally, we aimed to estimate preliminary effects on symptom severity and quality of life.

\section{Methods}

This is a non-randomized prospective patient preference study lasting 12 weeks comparing the intervention $(\mathrm{n}=20)$ with treatment as usual $(\mathrm{n}=20)$ in people with SMI. The intervention consisted of two lessons (Pilates and/or fitness) a week in a group of 4 - 6 participants via videoconferencing. Sport coaches interested to work with people with SMI were recruited from regular gyms and were trained by a psychiatrist and a psychomotor therapist to work with people with SMI.

\subsection{Participants}

Participants were recruited from the psychiatric outpatient facility of the University Medical Centre in Utrecht (The Netherlands). At this outpatient facility a diversity of patients is seen of which most patients suffer from SMI. We also recruited patients from a supported housing facility in Utrecht (Lister), where people receive outpatient mental healthcare while living on their own independently or in a facility with 24-hour care. Participants were recruited via their practitioners or mental health care worker and chose whether they want to participate in the intervention group or in the control group.

Inclusion criteria were SMI as defined as having a long lasting psychiatric disorder with impairments in social functioning needing psychiatric care, possessing a device with webcam and Internet connection and age between $16-70$. Exclusion criterion was being unable to exercise. The study design was reviewed by the medical ethical committee of the University Medical Centre in Utrecht (20-313/C) and informed consent of the participants was obtained after the procedures had been fully explained.

\subsection{Exercise Sessions}

Participants received a fitness mat at home to use during the sport lessons. Every 
lesson started with a welcome talk from the sport coach and experiences and questions of participants were discussed. At the end of the lesson there was also a brief moment to evaluate the lesson. The lessons were given at beginners level and the sport coach gave individual feedback to the participants and gave options to adjust the exercises when needed. The sport coaches were instructed to give the participants many positive feedbacks. Participants had the possibility to invite a friend, family member or mental health care worker to join the lessons. One hour prior to the lesson an email reminder was sent to the participants. If participants were absent for two lessons, the sport coach or researcher called the participant to ask for the reason of the absence and to offer help or give advice.

\subsection{Outcomes}

The primary outcome was feasibility measured at follow-up by a questionnaire based on the Measurement Instrument for Determinants of Innovations (MIDI) (Fleuren et al., 2014) and registered attendance. Secondary outcomes were symptom severity as measured at baseline and follow-up by the Brief Symptom Inventory (BSI) and quality of life as measured by the WHO Quality of Life questionnaire (WHOQOL-BREF) respectively. Additionally, data on age, sex, diagnosis and medication use were retrieved.

\subsection{Analysis}

Baseline characteristics were tested for between-group differences with Chi-square tests and t-test. Intention-to-treat analysis was done for all analyses. For the analysis of the MIDI we recoded negatively stated items according to the MIDI guideline (Fleuren et al., 2014) and used means, standard deviations and percentages for evaluation purposes. To test for between-group differences in within-group changes in health-related outcomes, we used Chi-square tests and t-tests. To test for between-group effects on health-related outcomes, we used linear regression adjusting for baseline differences between groups.

\section{Results}

20 participants were enrolled in the intervention group (10 Pilates, 8 fitness and 2 fitness/Pilates) and completed baseline measurement. 20 participants were enrolled in the control group, of which 3 were lost to follow-up at baseline measurement.

\subsection{Baseline Characteristics}

Baseline characteristics are shown in Table 1. $42.5 \%$ of the participants were female and the mean age was 41.8 . The majority of the participants had a psychotic spectrum disorder $(\mathrm{n}=23,57.5 \%), 9$ participants had a mood disorder (22.5\%), 2 participants had an autism spectrum disorder (5\%), 2 participants had a posttraumatic stress disorder (5\%) and one participant had a personality disorder (2.5\%). Groups only differed on medication use $(p=0.02)$, as more mood stabilizers were used in the treatment group. 
Table 1. Patient and disease characteristics and health-related outcomes for baseline and follow-up, including per protocol between-group analyses. Significant between-group differences $(p<0.05)$ are shown bold. WHOQOL-BREF $=$ WHO Quality of Life questionnaire). BSI = Brief Symptom Inventory, measured on a four point outcome scale. $\mathrm{B}=$ coefficient. $\mathrm{CI}=$ confidence interval. PTSS = post-traumatic stress disorder. M (SD) unless noted otherwise. $p$-value $<0.05$ is considered statistically significant.

\begin{tabular}{|c|c|c|c|c|c|c|c|c|c|}
\hline \multirow[b]{2}{*}{ Variable } & \multicolumn{3}{|c|}{ Intervention $(\mathrm{n}=20)$} & \multicolumn{3}{|c|}{ Treatment as usual $(\mathrm{n}=17)$} & \multicolumn{3}{|c|}{ Linear regression } \\
\hline & Baseline & 12 weeks & Change & Baseline & 12 weeks & Change & B & $95 \% \mathrm{CI}$ & $p$ \\
\hline Sex (female), n (\%) & $12(60.0)$ & & & $5(29.4)$ & & & & & 0.20 \\
\hline Age, $M(S D)$ & $44.3(13.6)$ & & & $39.3(12.1)$ & & & & & 0.25 \\
\hline Diagnoses, $\mathrm{n}(\%)$ & & & & & & & & & 0.11 \\
\hline Psychotic spectrum disorder & $11(55.0)$ & & & $12(70.6)$ & & & & & \\
\hline Mood disorder & $7(35)$ & & & $2(11.8)$ & & & & & \\
\hline Autism spectrum disorder & & & & $2(11.8)$ & & & & & \\
\hline PTSD & $2(10)$ & & & & & & & & \\
\hline Personality disorder & & & & $1(5.9)$ & & & & & \\
\hline \multicolumn{10}{|l|}{ Medication use, $\mathrm{n}(\%)$} \\
\hline Antipsychotics & $14(70.0)$ & & & $15(88.2)$ & & & & & 0.18 \\
\hline Antidepressants & $8(40.0)$ & & & $7(41.2)$ & & & & & 0.94 \\
\hline Benzodiazepines & $4(20.0)$ & & & $6(35.3)$ & & & & & 0.30 \\
\hline Mood stabilizers & $8(40.0)$ & & & $1(5.9)$ & & & & & 0.02 \\
\hline Non & $2(10.0)$ & & & $1(5.9)$ & & & & & 0.65 \\
\hline \multicolumn{10}{|l|}{ BSI, $M$} \\
\hline Sum score & 1.73 & 1.72 & -0.01 & 1.89 & 1.95 & 0.06 & -0.24 & $-0.65 / 0.16$ & 0.23 \\
\hline Psychoticism & 1.75 & 1.68 & -0.07 & 1.94 & 2.12 & 0.18 & -0.54 & $-1.12 / 0.04$ & 0.07 \\
\hline Paranoid thoughts & 1.81 & 1.78 & -0.03 & 1.91 & 2.09 & 0.19 & -0.39 & $-1.01 / 0.23$ & 0.21 \\
\hline \multicolumn{10}{|l|}{ WHOQOL-BREF, M } \\
\hline Domain I Physical health & 11.81 & 13.26 & 1.22 & 12.63 & 12.24 & -0.07 & 1.96 & $0.17 / 3.74$ & 0.03 \\
\hline Domain II Psychological & 12.04 & 12.91 & 0.98 & 12.26 & 11.89 & -0.37 & 1.34 & $-0.02 / 2.69$ & 0.05 \\
\hline Domain III Social relationships & 12.98 & 13.12 & 0.08 & 11.89 & 11.89 & 0.00 & 0.11 & $-2.03 / 2.25$ & 0.92 \\
\hline Domain IV Environment & 15.29 & 15.43 & 0.04 & 15.00 & 14.50 & -0.17 & 0.64 & $-0.47 / 1.75$ & 0.25 \\
\hline
\end{tabular}

\subsection{Dropout and Attendance Rate}

Participants dropped out after a single Pilates lesson due to lack of time $(\mathrm{n}=1)$ and due to technical problems $(\mathrm{n}=1)$ and after 11 fitness lessons because of self-doubt due to different strength levels in the group $(\mathrm{n}=1) .13$ participants attended at least $66 \%$ of the lessons. 13 participants continued with exercise after the intervention period.

\subsection{Feasibility}

$14(77.8 \%)$ of the participants were satisfied about exercise via video conferencing. Videoconferencing was easy to use for $14(77.8 \%)$ of the participants, although 10 participants (55.6\%) experienced technical difficulties. 12 participants (66.7\%) found it easy to exercise via videoconferencing. 10 participants (55.6\%) 
mentioned feeling less lonely and feeling physically fitter $(\mathrm{N}=14,77.8 \%)$ as advantages. Participants reported that exercise via video conferencing decreased the barrier to perform exercise because they could do the practise at home. One participant mentioned that the barrier to perform exercise decreased because she did not have to wear fancy sport clothes. Reported disadvantages were turning the camera on $(\mathrm{N}=5,27.8 \%)$ and having their personal space in the background $(\mathrm{N}=6,33.3 \%)$. One participant responded that she would recommend Pilates to everyone, but particularly to psychiatric patients.

\subsection{Symptom Severity and Quality of Life}

Results on symptom severity and quality of life are shown in Table 1. Linear regression showed significant improvement in physical $(p=0.03)$ and psychological quality of life $(p=0.05)$ in the intervention group compared to treatment as usual.

\section{Discussion}

Our study shows that Pilates and fitness via live videoconferencing are feasible for people with SMI during COVID-19 times. The majority of the participants found it easy to use and felt physically fit. The physical and psychological quality of life of the participants in the treatment group improved compared to treatment as usual.

Our results regarding feasibility are in line with previous research that investigated feasibility of exercise videoconferencing in patients with Down syndrome (Ptomey et al., 2018) and patients with heart failure (Hwang et al., 2017). The attendance rate in patients with Down syndrome was higher, likely because participants had a care giver that gave reminders. The dropout rate in our study was $15 \%$, which is lower than the average dropout rate of $26.7 \%$ that was found in a meta-analysis examining dropout rate in physical activity interventions in patients with schizophrenia (Vancampfort et al., 2016). This implicates that exercise via live videoconferencing is feasible for people with SMI and can be used as an alternative of face-to-face exercise or as a first step in motivating people with SMI to exercise.

Previous research (Skevington \& Wright, 2001) on the responsiveness of the WHOQOL-BREF in patients with a depression found a similar effect size and considered this a moderate effect. The improvement on quality of life can thus be considered clinically relevant. Together with the relatively low dropout, findings correspond with previous studies showing larger effects and lower dropout when exercise is supervised by qualified exercise professionals (Stubbs et al., 2018). Therefore, as with face-to-face sessions, we encourage exercise via videoconferencing to be supervised by qualified professionals who are trained to support people with SMI to overcome frequent (disease-related) barriers to exercise.

\section{Strengths and Limitations}

The biggest strength of our study is that it is, to the best of our knowledge, the 
first study examining the feasibility of exercise via videoconferencing for people with SMI. Such innovative interventions most likely ease access to supported physical activities and may help people with SMI to overcome barriers they often experience to engage in physical activities. Limitations of this study are caused by the non-randomized study design in which we only included 20 participants in each group. This affects the statistical power and most likely introduced selection bias. However, groups only differed in medication use and diagnoses were equally distributed among groups. Additionally, we did not include an active control group, e.g. a talk group, thus we did not determine whether the effect was caused by exercise or by social interaction.

In short, supervised group exercise via videoconferencing showed to be feasible for people with SMI in COVID-19 times. Improvements in physical and psychological quality of life were found compared to the control group. Further research in larger samples is needed to study the potential effect of exercise via videoconferencing in mitigating the negative effects of the COVID-19 pandemic on mental and physical health for people with SMI.

\section{Conflicts of Interest}

The authors declare no conflicts of interest regarding the publication of this paper.

\section{References}

Firth, J., Solmi, M., Wootton, R., Vancampfort, D., Schuch, F., Gilbody, S., Torous, J., Teasdale, S., Jackson, S., Smith, L., Eaton, M., Jacka, F., Veronese, N., Marx, W., Ashdown-Franks, G., Siskind, D., Sarris, J., Rosenbaum, S., Carvalho, A., \& Stubbs, B. (2020). A Meta-Review of "Lifestyle Psychiatry": The Role of Exercise, Smoking, Diet and Sleep in the Prevention and Treatment of Mental Disorders. World Psychiatry, 19, 360-380. https://doi.org/10.1002/wps.20773

Fleuren, M. A. H., Paulussen, T. G. W. M., Dommelen, P., \& Van Buuren, S. (2014). Towards a Measurement Instrument for Determinants of Innovations. International Journal for Quality in Health Care, 26, 501-510. https://doi.org/10.1093/intqhc/mzu060

Hwang, R., Mandrusiak, A., Morris, N. R., Peters, R., Korczyk, D., Bruning, J., \& Russell, T. (2017). Exploring Patient Experiences and Perspectives of a Heart Failure Telere Habilitation Program: A Mixed Methods Approach. Heart and Lung: Journal of Acute and Critical Care, 46, 320-327. https://doi.org/10.1016/j.hrtlng.2017.03.004

O’Connor, R. C., Wetherall, K., Cleare, S., McClelland, H., Melson, A. J., Niedzwiedz, C. L., O’Carroll, R. E., O’Connor, D. B., Platt, S., Scowcroft, E., Watson, B., \& Zorte, T. (2020). Mental Health and Wellbeing during the COVID-19 Pandemic: Longitudinal Analyses of Adults in the UK COVID-19 Mental Health \& Wellbeing Study. British Journal of Psychiatry, 1-17.

Ptomey, L. T., Szabo, A. N., Willis, E. A., Greene, J. L., Danon, J. C., Washburn, R. A., Forsha, D. E., \& Donnelly, J. E. (2018). Remote Exercise for Adults with Down Syndrome. Translational Journal of the American College of Sports Medicine, 3, 60-65.

Skevington, S. M., \& Wright, A. (2001). Changes in the Quality of Life of Patients Receiving Antidepressant Medication in Primary Care: Validation of the WHOQOL-100. British Journal of Psychiatry, 178, 261-267. https://doi.org/10.1192/bjp.178.3.261 
Stanton, R., To, Q. G., Khalesi, S., Williams, S. L., Alley, S. J., Thwaite, T. L., Fenning, A. S., \& Vandelanotte, C. (2020). Depression, Anxiety and Stress during COVID-19: Associations with Changes in Physical Activity, Sleep, Tobacco and Alcohol Use in Australian Adults. International Journal of Environmental Research and Public Health, 17, 4065. https://doi.org/10.3390/ijerph17114065

Stubbs, B., Vancampfort, D., Hallgren, M., Firth, J., Veronese, N., Solmi, M., Brand, S., Cordes, J., Malchow, B., Gerber, M., Schmitt, A., Correll, C. U., De Hert, M., Gaughran, F., Schneider, F., Kinnafick, F., Falkai, P., Moller, H.-J., \& Kahl, K. G. (2018). EPA Guidance on Physical Activity as a Treatment for Severe Mental Illness: A Meta-Review of the Evidence and Position Statement from the European Psychiatric Association (EPA), Supported by the International Organization of Physical Therapists in Mental. European Psychiatry: The Journal of the Association of European Psychiatrists, 54, 124-144. https://doi.org/10.1016/j.eurpsy.2018.07.004

Vancampfort, D., Firth, J., Schuch, F. B., Rosenbaum, S., Mugisha, J., Hallgren, M., Probst, M., Ward, P. B., Gaughran, F., De Hert, M., Carvalho, A. F., \& Stubbs, B. (2017). Sedentary Behavior and Physical Activity Levels in People with Schizophrenia, Bipolar Disorder and Major Depressive Disorder: A Global Systematic Review and Meta-Analysis. World Psychiatry: Official Journal of the World Psychiatric Association (WPA), 16, 308-315. https://doi.org/10.1002/wps.20458

Vancampfort, D., Rosenbaum, S., Schuch, F. B., Ward, P. B., Probst, M., \& Stubbs, B. (2016). Prevalence and Predictors of Treatment Dropout from Physical Activity Interventions in Schizophrenia: A Meta-Analysis. General Hospital Psychiatry, 39, 15-23. https://doi.org/10.1016/j.genhosppsych.2015.11.008

Vindegaard, N., \& Benros, M. E. (2020). COVID-19 Pandemic and Mental Health Consequences: Systematic Review of the Current Evidence. Brain, Behavior, and Immunity, 89, 531-542. https://doi.org/10.1016/j.bbi.2020.05.048 